https://helda.helsinki.fi

\title{
Generalization of Taylor's formula to particles of arbitrary inertia
}

\author{
Boi, Simone
}

2018-10-02

Boi , S , Mazzino , A , Muratore-Ginanneschi , P \& Olivieri , S 2018 , ' Generalization of

Taylor's formula to particles of arbitrary inertia ', Physical Review Fluids , vol. 3 , no. 10 , 104501 . https://doi.org/10.1103/PhysRevFluids.3.104501

http://hdl.handle.net/10138/247104

https://doi.org/10.1103/PhysRevFluids.3.104501

unspecified

publishedVersion

Downloaded from Helda, University of Helsinki institutional repository.

This is an electronic reprint of the original article.

This reprint may differ from the original in pagination and typographic detail.

Please cite the original version. 


\title{
Generalization of Taylor's formula to particles of arbitrary inertia
}

\author{
S. Boi, ${ }^{1}$ A. Mazzino, ${ }^{2,3}$ P. Muratore-Ginanneschi, ${ }^{1}$ and S. Olivieri ${ }^{2}$ \\ ${ }^{1}$ Department of Mathematics and Statistics, University of Helsinki, \\ Gustaf Hällströmin katu 2b, Helsinki, Finland \\ ${ }^{2}$ DICCA, University of Genova, Via Montallegro 1, 16145 Genova, Italy \\ ${ }^{3}$ INFN, Genova Section, Via Dodecaneso 33, 16146 Genova, Italy
}

(Received 3 January 2018; published 2 October 2018)

\begin{abstract}
One of the cornerstones of turbulent dispersion is the celebrated Taylor's formula. This formula expresses the rate of transport (i.e., the eddy diffusivity) of a tracer as a time integral of the fluid velocity autocorrelation function evaluated along the fluid particle trajectories. Here, we review the hypotheses which permit us to extend Taylor's formula to particles of any inertia. The hypotheses are independent of the details of the inertial particle model. We also show by explicit calculation that the hypotheses encompass cases when memory terms such as Basset's and Faxén's corrections are taken into account in the modeling of inertial particle dynamics.
\end{abstract}

DOI: 10.1103/PhysRevFluids.3.104501

\section{INTRODUCTION}

In the early 1920s, Taylor derived what can be fairly considered one of the cornerstones of largescale transport of tracer particles in fluid flows [1]. Tracer particles are small particles not affecting the carrier velocity field with their motion:

$$
\frac{d \boldsymbol{x}}{d t}=\boldsymbol{u}(\boldsymbol{x}, t)
$$

In the limit of long observation time and under rather general assumptions, Taylor observed that the mean square of tracer particle displacement grows linearly in time with a rate now usually referred to as the eddy-diffusivity coefficient (see, e.g., Refs. [2-7]):

$$
\left\langle\|\boldsymbol{x}(t)-\langle\boldsymbol{x}(t)\rangle\|^{2}\right\rangle \sim 2 D t .
$$

Based on this observation, Taylor established a first principle identity expressing the tracer particle eddy diffusivity as a time integral of the fluid velocity autocorrelation function evaluated along the fluid trajectories:

$$
D=\lim _{t \rightarrow \infty} \int_{0}^{t} d s\langle\delta \boldsymbol{u}(\boldsymbol{x}(t), t) \cdot \delta \boldsymbol{u}(\boldsymbol{x}(s), s)\rangle,
$$

where $\delta \boldsymbol{u}(\boldsymbol{x}(t), t)=\boldsymbol{u}(\boldsymbol{x}(t), t)-\langle\boldsymbol{u}(\boldsymbol{x}(t), t)\rangle$.

Since then, the relation (3) now going under the name of Taylor's formula has played a key role in the analysis of turbulent dispersion of tracers [8,9]. We refer, e.g., to Chapter 12 of the textbook [10] for a review, including an introduction to the vast existing literature.

Tracer dispersion is a small subset of a much larger class of transport problems: the transport of inertial particles. Inertial particles are small particles having a finite size and/or density different from that of the carrier fluid [11]. Inertial particles are encountered practically everywhere, from our atmosphere (e.g., affecting the global radiative budget of the Earth's climate system by scattering and absorbing long-wave and short-wave radiation [12], or leading to increased droplet collisions 
and the formation of larger droplets with a key role for rain initiation [13-15]) and ocean (e.g., in relation to phytoplankton dynamics in turbulent ocean [16]) to astrophysics (in relation to planet formation, e.g., Refs. [17,18]).

It is therefore not surprising that deriving extensions of Taylor's formula to inertial particle dynamics has been stirring interest for half a century $[19,20]$. A review of early results can be found in Ref. [21]. Furthermore, most of the existing analytic investigations of inertial particles (e.g., Refs. [22-25]; see also Ref. [26] for further references) use Taylor's formula as a key ingredient. These works often set out to derive methods for iteratively solving coupled systems equations governing the fluid Eulerian and Lagrangian correlation functions.

Our aim here is to review in a fashion independent of model details the conditions presiding over the expression of the inertial particle eddy diffusivity as an integral of the correlation functions of fluid velocity and external forces evaluated along the particle trajectories.

There are two closely intertwined reasons why we think that this is interesting.

First, the past decades have seen major developments in the experimental techniques to measure Eulerian fluid flows under real conditions. An outstanding illustration of this fact is the reconstruction of sea surface currents as spatiotemporal fields via high-frequency radar (see, e.g., Ref. [27]). Once the Eulerian carrier field is known, the extension of Taylor's formula to inertial particles becomes a powerful tool to extricate physical information. The reason is that the formula allows us to infer the qualitative properties of the eddy diffusivity from the space structure of the Eulerian field. By way of example, a field having closed structures (i.e., rolls) is expected to trap light particles, thus causing a reduction of the transport with respect to flows with open streamlines. This observation in the tracer case is instrumental in predicting the so-called constructive and destructive interference regimes [28]. The generalization of Taylor's formula thus opens the way to the investigation of new links between the dynamics and the statistics of inertial particles.

Similar arguments also apply to external forces, which are functions depending-even nonlinearly — on the carrier field itself or the particle trajectories explicitly. This brings us to the second reason for this work. The exact form and relative importance of the forces exerted on inertial particles has indeed been object of controversy since the work [20]. In more recent years, a consensus seems to have been reached based on the first principle analysis of Ref. [29] and the inclusion of the correction term advocated in Refs. [30,31]. An overview of the reasons motivating the development of such models is provided by Ref. [32]. Nevertheless, an analysis independent of model details is justified as it provides a framework to assess the relative importance for diffusion of the correction terms distinguishing models of inertial particle dynamics.

Thanks to our generalized Taylor formula, one can evaluate the autocorrelations and the cross correlations of flow and external forces, either through available data or from analytical dynamical models. This allows investigating how and in what regions of the flow the model terms and their mutual interactions contribute to transport, providing more physical information about the problem. Moreover, whenever an analytical calculation of the trajectories is available, it becomes possible to compute exactly the variation of the eddy diffusivity caused by external forces and correction terms of the dynamical model. By way of example, we will consider the effect of Coriolis, Lorentz, Faxén, and lift forces, and in some simple cases we will see how these forces can increase or decrease asymptotic transport, even hindering the molecular diffusion.

Combining the generalized Taylor formula with autocorrelations and cross correlations of the carrier field and external forces therefore renders it possible to evaluate the response of the eddy diffusivity to variations of the external stirring and the introduction of correction terms in the dynamical model. In this sense, the generalized Taylor formula represents an extension of fluctuation-dissipation-type relations (see, e.g., Ref. [33]) to particle dynamics in fluids.

The paper is organized as follows: In Sec. II, we analyze the hypotheses leading to the generalization of Taylor's formula to a wide class of models of inertial particle dynamics. Technical aspects of this analysis are deferred to an Appendix. An important advantage of a model-detail independent derivation is to ease the inclusion of the effect of external forces in generalized Taylor's formula. We avail ourselves of this fact to analyze specific models of inertial particle transport. 
In Sec. III, we apply the general result to the Basset-Boussinesq-Oseen model, by considering several dynamic scenarios relevant for applications. In Sec. IV, we derive Taylor's formula for the Maxey-Riley model. This is a refinement of the expression obtained in Ref. [22], which retained only leading orders in the expansion in powers of the Stokes number. Inclusion of lift forces in dynamical models is treated in Sec. V and, finally, conclusions are presented in Sec. VI.

\section{GENERALIZED TAYLOR'S FORMULA FOR INERTIAL PARTICLE TRANSPORT}

We consider a general model of mutually noninteracting inertial particles in a carrier flow. The state of a single inertial particle is specified by its position $\xi(t)$ and velocity $\boldsymbol{v}(t)$ at time $t$. We denote by $\boldsymbol{u}$ the carrier flow, a vector field joint function of space and time variables. We suppose that the dynamics is amenable to the form of a system of integrodifferential equations in $d$-spatial dimensions, where the idiosyncratic characteristics of the model are encapsulated in kernels $\mathbb{K}_{i}, i=$ $0, \ldots, N$ :

$$
\begin{aligned}
& \dot{\boldsymbol{\xi}}(t)=\boldsymbol{v}(t), \\
& \boldsymbol{v}(t)=\boldsymbol{\sigma}(\boldsymbol{\xi}(0), \boldsymbol{v}(0), t)+\int_{0}^{t} d s \mathbb{K}_{0}(t-s) \boldsymbol{u}(\boldsymbol{\xi}(s), s)+\sum_{i=1}^{N} \int_{0}^{t} d s \mathbb{K}_{i}(t-s) \boldsymbol{f}_{i}(\boldsymbol{\xi}(s), s) .
\end{aligned}
$$

Most inertial particle models in the literature are amenable to the form (4b), with possibly the only notable exception of angular dynamics models (see, e.g., Ref. [34]). In what follows, we suppose that the transient term $\sigma(\xi(0), v(0), t)$ depending on the initial conditions does not play any role in the asymptotic diffusion and can be for this reason neglected. All specific models herein considered fulfill this assumption. We also expect that under general physical conditions the statistics of inertial particles attains a time asymptotic stationary diffusive regime independent of initial conditions (see also Hypothesis III below).

The vectors $f_{i} i=1, \ldots, N$ stand for external forces per unit mass acting on the particle such as the buoyancy and the Brownian and Coriolis forces. The detailed form of the $d \times d$-real-matrixvalued integral kernels $\mathbb{K}_{0}$ and $\mathbb{K}_{i}$ is not important for the analysis of the current section. Drawing on Ref. [19] we, however, require that

Hypothesis $I$. The integral kernels are stationary and have absolutely integrable components

$$
\int_{0}^{\infty} d t\left|\mathbb{K}_{i}^{m n}(t)\right|<K_{\star}<\infty, \quad \forall m, n=1, \ldots, d, \quad \text { and } \quad \forall i=0, \ldots, N .
$$

The hypothesis implies the existence of the Fourier-Laplace transforms

$$
\hat{\mathbb{K}}_{i}(z)=\int_{0}^{\infty} d t e^{-z t} \mathbb{K}_{i}(t), \quad \operatorname{Re} z>0, \quad i=0, \ldots, N .
$$

Our aim is to compute the inertial particle eddy-diffusivity tensor

$$
\mathrm{D}=\lim _{t \uparrow \infty} \frac{1}{2 t}\langle(\xi(t)-\langle\boldsymbol{\xi}(t)\rangle) \otimes(\boldsymbol{\xi}(t)-\langle\boldsymbol{\xi}(t)\rangle)\rangle .
$$

In Eq. (6), the symbol $\otimes$ denotes the tensor product of vectors and $\langle\cdots\rangle$ stands for an ensemble average. Ensemble average means here average over any source of randomness in the model (e.g., initial data, parameter uncertainty, or random carrier velocity field). By (4a), we can always couch the eddy diffusivity into the equivalent form

$$
\mathbb{D}=\lim _{t \uparrow \infty} \operatorname{Sym} \int_{0}^{t} d s\langle\delta \boldsymbol{v}(t) \otimes \delta \boldsymbol{v}(s)\rangle
$$

where

$$
\delta \boldsymbol{v}(t) \equiv \boldsymbol{v}(t)-\langle\boldsymbol{v}(t)\rangle
$$


and Sym stands for the tensor symmetrization operation

$$
\operatorname{Sym}\langle\delta \boldsymbol{v}(t) \otimes \delta \boldsymbol{v}(s)\rangle=\frac{\langle\delta \boldsymbol{v}(t) \otimes \delta \boldsymbol{v}(s)+\delta \boldsymbol{v}(s) \otimes \delta \boldsymbol{v}(t)\rangle}{2} .
$$

The qualitative reason why the eddy diffusivity is an important indicator of particle motion is given by the central limit theorem [1]. If the particle velocity autocorrelation function decays sufficiently quickly, $\boldsymbol{\xi}(t)$ becomes approximately Gaussian for large times with variance characterized by the eddy diffusivity tensor (6). However, it should be recalled that the existence of a finite limit for (6) is not always granted. There are physical systems for which $\mathbb{D}$ may vanish (subdiffusion) or diverge (superdiffusion); see, e.g., Ref. [35].

Here we do not assume directly the existence of (6) but we aim to derive it as a consequence of hypotheses made at the level of the second-order statistics of the carrier velocity field and the external forces evaluated along particle trajectories.

We start by defining the set of Lagrangian $d \times d$-matrix-valued correlation functions

$$
\tilde{\mathbb{C}}_{i j}\left(t, t^{\prime}\right)=\left\langle\boldsymbol{\phi}_{i}(\xi(t), t) \otimes \boldsymbol{\phi}_{j}\left(\xi\left(t^{\prime}\right), t^{\prime}\right)\right\rangle, \quad i, j=0, \ldots, N
$$

where

$$
\phi_{i}(\xi(t), t)= \begin{cases}\boldsymbol{u}(\boldsymbol{\xi}(t), t)-\langle\boldsymbol{u}(\boldsymbol{\xi}(t), t)\rangle & \text { if } i=0, \\ \boldsymbol{f}_{i}(\boldsymbol{\xi}(t), t)-\left\langle\boldsymbol{f}_{i}(\boldsymbol{\xi}(t), t)\right\rangle & \text { if } i=1, \ldots, N .\end{cases}
$$

Upon recalling (4b), it is straightforward to verify that

$$
\operatorname{Sym} \int_{0}^{t} d s\langle\delta \boldsymbol{v}(t) \otimes \delta \boldsymbol{v}(s)\rangle=\sum_{i j=0}^{N} \operatorname{Sym} \int_{0}^{t} d s_{3} \int_{0}^{t} d s_{1} \int_{0}^{s_{1}} d s_{2} \mathbb{K}_{i}\left(t-s_{3}\right) \tilde{\mathbb{C}}_{i j}\left(s_{3}, s_{2}\right) \mathbb{K}_{j}^{T}\left(s_{1}-s_{2}\right) .
$$

The superscript $T$ denotes here and below the matrix transposition operation.

As a second step, we require that the correlation functions satisfy suitable integrability conditions. Specifically, we suppose that

Hypothesis II. There exists a positive-definite scalar function $F$ such that for any $t, t^{\prime}$

$$
\left|\tilde{\mathbb{C}}_{i j}^{m n}\left(t, t^{\prime}\right)\right|<F\left(t-t^{\prime}\right) \quad \forall m, n=1, \ldots, d, \quad \text { and } \quad \forall i, j=0, \ldots, N
$$

with $F(t)=F(-t)$ and

$$
\int_{0}^{\infty} d t F(t)=F_{\star}<\infty .
$$

In words, we are hypothesizing that Lagrangian correlations decay sufficiently fast to take limits under the multiple integral sign. This is important because in the appendix we show that for any finite $t$

$$
\operatorname{Sym} \int_{0}^{t} d s\langle\delta \boldsymbol{v}(t) \otimes \delta \boldsymbol{v}(s)\rangle=\sum_{i j=0}^{N} \operatorname{Sym} \int_{0}^{t} d s_{3} \int_{0}^{t} d s_{1} \int_{0}^{s_{1}} d s_{2} \mathbb{K}_{i}\left(s_{3}\right) \tilde{\mathbb{C}}_{i j}\left(t-s_{3}, t-s_{2}\right) \mathbb{K}_{j}^{T}\left(s_{1}\right) .
$$

We thus set the scene to introduce our last hypothesis. We posit that

Hypothesis III. All the Lagrangian correlation functions (8) have a well-defined stationary limit

$$
\mathbb{C}_{i j}(t)=\lim _{t^{\prime} \uparrow \infty} \tilde{\mathbb{C}}_{i j}\left(t+t^{\prime}, t^{\prime}\right) .
$$

An immediate consequence of the definition (8) and of Hypothesis III is that for any finite $t$

$$
\mathbb{C}_{i j}(t)=\lim _{t^{\prime} \uparrow \infty} \tilde{\mathbb{C}}_{j i}^{T}\left(t^{\prime}, t^{\prime}+t\right)=\lim _{t^{\prime} \uparrow \infty} \tilde{\mathbb{C}}_{j i}^{T}\left(t^{\prime}-t, t^{\prime}\right)=\mathbb{C}_{j i}^{T}(-t) .
$$


In the Appendix, we combine hypotheses I-III to show that Taylor's identity holds true in the generalized form

$$
\mathbb{D}=\sum_{i, j=0}^{N} \hat{\mathbb{K}}_{i}(0) \frac{\hat{\mathbb{C}}_{i j}(0)+\hat{\mathbb{C}}_{j i}^{T}(0)}{2} \hat{\mathbb{K}}_{j}^{T}(0)=\sum_{i, j=0}^{N} \hat{\mathbb{K}}_{i}(0) \int_{0}^{\infty} d s \frac{\mathbb{C}_{i j}(s)+\mathbb{C}_{j i}^{T}(s)}{2} \hat{\mathbb{K}}_{j}^{T}(0)
$$

with

$$
\hat{\mathbb{C}}_{i j}(z)=\int_{0}^{\infty} d t e^{-z t} \mathbb{C}_{i j}(t), \quad \operatorname{Re} z>0 .
$$

A few remarks on the nature of the hypotheses are in order.

The validity of hypothesis I can be checked a priori from the explicit form of the equation of motion of inertial particle models.

Hypotheses II and III are instead not obviously granted. Their validity is an assumption on the properties of the solutions of (4). From the physics slant, we need hypothesis II to control memory effects. For example, relaxation dynamics of infinite dimensional systems with Boltzmann equilibrium may give rise to ageing phenomena [36]. Similar very slow decay of Lagrangian correlations must be ruled out in order to apply the dominated convergence theorem which we need to generalize Taylor's formula.

Eulerian carrier velocity field and external forces are in general explicit functions of the time variable. Lagrangian correlation functions may become asymptotically stationary (hypothesis III) in consequence of the ensemble average operation $\langle\cdots\rangle$. For example, hypotheses II and III are satisfied if the Eulerian statistics of velocity field is a random Gaussian ensemble $\delta$ correlated in time, a widely applied stylized model of a turbulent field [37].

Finally, the foregoing hypotheses are essentially the same as those underlying the derivation of Green-Kubo formulas [38] in nonequilibrium statistical mechanics. It is in this sense justified to regard Taylor's formula as an hydrodynamic counterpart of these relations.

\section{BASSET-BOUSSINESQ-OSEEN MODEL}

We now turn to apply the general results of Sec. II to explicit models of dynamics. To start with, let us consider the simplest and oldest model for inertial particles in an incompressible flow, the so-called Basset-Boussinesq-Oseen equation [20]:

$$
\frac{d \boldsymbol{v}}{d t}(t)=\frac{\boldsymbol{u}(\boldsymbol{\xi}(t), t)-\boldsymbol{v}(t)}{\tau}+\beta \frac{d \boldsymbol{u}}{d t}(\boldsymbol{\xi}(t), t)+\boldsymbol{f}+\sqrt{\frac{3 \beta}{\pi \tau}} \int_{0}^{t} \frac{d s}{\sqrt{t-s}} \frac{d}{d s}[\boldsymbol{u}(\boldsymbol{\xi}(s), s)-\boldsymbol{v}(s)] .
$$

In the above equation, $\boldsymbol{u}$ is the carrier field, and the pressure gradient term is estimated as $\nabla p \propto$ $-d \boldsymbol{u} / d t$ [20], where

$$
\frac{d \boldsymbol{u}}{d t}(\boldsymbol{\xi}(t), t) \equiv\left[\partial_{t} \boldsymbol{u}(\boldsymbol{x}, t)+\boldsymbol{v}(t) \cdot \nabla \boldsymbol{u}(\boldsymbol{x}, t)\right]_{\boldsymbol{x}=\boldsymbol{\xi}(t)} .
$$

The term $\boldsymbol{f}$ is a generic external force per unit mass, $\tau \equiv r_{p}^{2} /(3 v \beta)$ denotes the Stokes time, with $r_{p}$ being the radius of inertial particles (supposed to be spherical) and $v$ being the fluid kinematic viscosity. Finally, the parameter $\beta$ is the added-mass factor, $\beta \equiv 3 \rho_{f} /\left(\rho_{f}+2 \rho_{p}\right) \in[0,3]$ built from the constant fluid density $\rho_{f}$ and the particle density $\rho_{p}$. Equation (16) requires no-slip condition on the particle surface. This equation was initially introduced as a model of a particle carried by a static and uniform field $\boldsymbol{u}$. It was subsequently realized that (16) still holds for the dynamics of particles carried by nonuniform and time-dependent velocity fields, under the following assumptions [20]. First, particles must be very small so that any $o\left(r_{p} / L\right)$ effect is negligible, where $L$ is the minimal variation length of the flow. Second, the particle Reynolds number $\operatorname{Re}_{p}=(\max |\boldsymbol{u}-\boldsymbol{v}|) r_{p} / v$ must be sufficiently close to 0 . Finally, the Stokes number, that is, the ratio between Stokes time and the 
smallest advection time $\tau_{F}$ in the flow, must satisfy $\tau / \tau_{F} \ll 1$. Under these hypotheses, Eq. (16) is within leading-order accuracy in the aforementioned parameters equivalent to the Maxey-Riley model [29].

The integral on the right-hand side of (16) is the Basset history term describing the force due to the lagging boundary layer development with changing relative velocity of the particle moving through the fluid, under the condition $\boldsymbol{v}(0)=\boldsymbol{u}(\boldsymbol{\xi}(0), 0)$ [39]. If this latter condition is not satisfied, a correction term must be added [40] so that the Laplace transform of the corrected equation coincides with that obtained from Eq. (16). For our purposes, it is therefore sufficient to use Eq. (16).

\section{A. The buoyancy-forced case}

\section{Constant force}

If the external force describe solely buoyancy, i.e., $f=(1-\beta) g$ [41], the Fourier-Laplace transform of Eq. (16) yields

$$
\hat{\boldsymbol{v}}(z)=\frac{(1-\beta) \boldsymbol{u}(\boldsymbol{\xi}(0), 0)}{a(z)}+\frac{(\beta-1) z+a(z)}{a(z)} \hat{\boldsymbol{u}}(z)+\frac{(1-\beta) \boldsymbol{g}}{z a(z)},
$$

where

$$
\hat{\boldsymbol{v}}(z) \equiv \int_{0}^{\infty} d t e^{-z t} \boldsymbol{v}(t)
$$

and

$$
\hat{\boldsymbol{u}}(z) \equiv \int_{0}^{\infty} d t e^{-z t} \boldsymbol{u}(\boldsymbol{\xi}(t), t)
$$

and finally

$$
a(z)=z+\frac{1}{\tau}+\sqrt{\frac{3 \beta z}{\tau}} .
$$

If we contrast Eq. (17) with the Fourier-Laplace transform of Eq. (4b), we find

$$
\hat{\boldsymbol{v}}(z)=\hat{\boldsymbol{\sigma}}(\boldsymbol{\xi}(0), \boldsymbol{v}(0), z)+\hat{\mathbb{K}}_{0}(z) \hat{\boldsymbol{u}}(z)+\sum_{i=1}^{N} \hat{\mathbb{K}}_{i}(z) \hat{\boldsymbol{f}}_{i}(z)
$$

with

$$
\begin{aligned}
\hat{\mathbb{K}}_{0}(z) & =\hat{K}_{0}(z) \mathbb{1}=\frac{(\beta-1) z+a(z)}{a(z)} \mathbb{1}, \\
\hat{\mathbb{K}}_{1}(z) & =\hat{K}_{1}(z) \mathbb{1}=\frac{1}{a(z)} \mathbb{1}, \\
\hat{\boldsymbol{\sigma}}(\boldsymbol{\xi}(0), \boldsymbol{v}(0), z) & =(1-\beta) \boldsymbol{u}(\boldsymbol{\xi}(0), 0) \hat{K}_{1}(z),
\end{aligned}
$$

satisfying $\hat{K}_{0}(0)=1$ and $\hat{K}_{1}(0)=\tau$.

An elementary calculation shows that the transient term does not contribute to the eddy diffusivity. Upon neglecting the transient, the inverse Fourier-Laplace transform of Eq. (19) yields the explicit form of Eq. (4b),

$$
\boldsymbol{v}(t)=\int_{0}^{t} d s K_{0}(t-s) \boldsymbol{u}(s)+(1-\beta) \boldsymbol{g} \int_{0}^{t} d s K_{1}(s) .
$$

By virtue of Eq. (14), we recover Taylor's 1921 formula

$$
\mathbb{D}=\operatorname{Sym} \hat{\mathbb{C}}_{00}(0)=\lim _{t \uparrow \infty} \operatorname{Sym} \int_{0}^{t} d s\langle\delta \boldsymbol{u}(\boldsymbol{\xi}(s), s) \otimes \delta \boldsymbol{u}(\boldsymbol{\xi}(t), t)\rangle,
$$

and the trace of the resulting eddy-diffusivity tensor is the same as in Ref. [20]. 


\section{Brownian force}

We can repeat the same steps as above in the presence of an external Brownian force per unit mass equal to $\sqrt{2 D_{0}} / \tau \boldsymbol{\eta}(t)$, with $\boldsymbol{\eta}(t)$ being a white-noise process coupled by a constant molecular diffusivity $D_{0}$ [25]. The equation for the particle velocity can be couched (neglecting transient terms) into the form (4b)

$$
\boldsymbol{v}(t)=\int_{0}^{t} d s K_{0}(t-s) \boldsymbol{u}(z)+\int_{0}^{t} d s K_{1}(t-s)\left[(1-\beta) \boldsymbol{g}+\frac{\sqrt{2 D_{0}}}{\tau} \eta(s)\right] .
$$

In this case, we define

$$
\boldsymbol{\phi}_{i}(\xi(t), t)= \begin{cases}\boldsymbol{u}(\boldsymbol{\xi}(t), t)-\langle\boldsymbol{u}(\boldsymbol{\xi}(t), t)\rangle & \text { if } i=0, \\ \frac{\sqrt{2 D_{0}}}{\tau} \boldsymbol{\eta}(s) & \text { if } i=1 .\end{cases}
$$

We obtain

$$
\begin{aligned}
& \mathbb{C}_{00}(t)=\lim _{t^{\prime} \uparrow \infty} \tilde{\mathbb{C}}_{00}\left(t+t^{\prime}, t^{\prime}\right)=\lim _{t^{\prime} \uparrow \infty}\left\langle\delta \boldsymbol{u}\left(\xi\left(t+t^{\prime}\right), t+t^{\prime}\right) \otimes \delta \boldsymbol{u}\left(\boldsymbol{\xi}\left(t^{\prime}\right), t^{\prime}\right)\right\rangle, \\
& \mathbb{C}_{11}(t)=\lim _{t^{\prime} \uparrow \infty} \tilde{\mathbb{C}}_{11}\left(t+t^{\prime}, t^{\prime}\right)=\frac{2 D_{0}}{\tau^{2}} \delta(t) .
\end{aligned}
$$

Furthermore, $\mathbb{C}_{10}(t)=\mathbb{C}_{01}(t)=0$ because of the causal independence between white noise $\eta(t)$ and fluid velocity $\boldsymbol{u}\left(t^{\prime}\right)$ at time $t^{\prime}<t$. Upon recalling the identity $\int_{0}^{\infty} d t \delta(t)=1 / 2$ and Eq. (14), we obtain for inertial particles an expression of the eddy diffusivity equal in form to that of tracer particles

$$
\mathbb{D}=D_{0} \mathbb{1}+\operatorname{Sym} \hat{\mathbb{C}}_{00}(0) .
$$

It must be, however, emphasized that Eq. (26) must be evaluated along trajectories which differ for inertial particles from those of tracer particles.

\section{B. Inclusion of the Lorentz force}

A generalized form of Taylor's formula is possible if inertial particles are subject to a Lorentz force $-q \boldsymbol{B} \times \boldsymbol{v}$ in a constant magnetic field $\boldsymbol{B}$ and interparticle interactions are neglected [42]. This can be regarded as a stylized model of charged particles in a plasma [43-45]. Furthermore, it is possible to show that when in a solid the electron-electron collision mean-free path is far smaller than the system width, electrons can be modeled as a fluid where mutual collisions are taken into account by viscous dissipation [46].

The Laplace transform of the equation of motion without transient yields

$$
\hat{\mathbb{A}}(z) \hat{\boldsymbol{v}}(z)=[(\beta-1) z+a(z)] \hat{\boldsymbol{u}}(z)+\frac{\sqrt{2 D_{0}}}{\tau} \hat{\boldsymbol{\eta}}(z),
$$

where we defined the strictly positive definite tensor $\hat{A}(z)$ with components

$$
\hat{\mathrm{A}}^{\mu \nu}(z)=a(z) \delta^{\mu \nu}+\gamma B^{\nu} \epsilon^{\mu \sigma \nu},
$$

where $a$ is defined by (18) and

$$
\gamma=\frac{q}{\frac{4}{3} \pi r_{p}^{3} \rho_{p}}
$$

Upon inverting $\hat{A}(z)$, we obtain an equation of the form (19), whence it is straightforward to derive generalized Taylor's formula

$$
\mathbb{D}=\frac{D_{0}}{\tau^{2}} \hat{\mathbb{A}}^{-1}(0)\left(\hat{\mathbb{A}}^{-1}\right)^{T}(0)+\frac{1}{\tau^{2}} \hat{\mathbb{A}}^{-1}(0) \operatorname{Sym}\left(\hat{\mathbb{C}}_{00}(0)\right)\left(\hat{\mathbb{A}}^{-1}\right)^{T}(0)
$$


with

$$
\left(\hat{\mathrm{A}}^{-1}\right)^{\mu \nu}(z)=\frac{1}{a^{2}(z)+\gamma^{2}\|\boldsymbol{B}\|^{2}}\left[a(z) \delta^{\mu \nu}-\gamma B^{i} \epsilon^{\mu \sigma \nu}+\frac{\gamma^{2}}{a(z)} B^{k} B^{j}\right] .
$$

Notice that due to the Laplace transform on Eq. (27), the transformed Green's function $\left(\hat{\mathrm{A}}^{-1}\right)^{\mu \nu}(z)$ is dimensionally a time, and consistently Eq. (29) has the same dimensions of $D_{0}$.

\section{Limit of vanishing carrier velocity field}

A simple application is when $\boldsymbol{u}=0$ in $d=3$ and the magnetic field $\boldsymbol{B}$ is oriented along the third coordinate axis ( $\boldsymbol{B}=B \boldsymbol{e}_{3}$ for $\boldsymbol{e}_{3}$ is the unit vector spanning the axis). We get

$$
\mathrm{D}=\operatorname{diag}\left(\frac{D_{0}}{1+\gamma^{2} B^{2} \tau^{2}}, \frac{D_{0}}{1+\gamma^{2} B^{2} \tau^{2}}, D_{0}\right),
$$

where we can observe a reduction of the transport due to the action of the magnetic field. Equation (30) generalizes the result of Ref. [45], by showing that the added mass effect and the Basset history term do not play any role in the asymptotic transport when the fluid is at rest and a Lorenz force is present. This result is also in agreement with Refs. [47,48], where it is shown that in still fluids, the Stokes drag term and Basset force generate a noise term with memory which, however, does not affect the eddy diffusivity. On the other hand, there is much investigation in literature about strong differences Basset history term can make in particle motion when the fluid is not at rest. One of the most representative cases is Ref. [49]. Therein, it is shown that in a cellular flow inertial particles with small $\tau$ remain trapped into cells (i.e., no diffusion), whereas Basset history force term lets them escape along the cell separatrixes, resulting in oscillating ballistic trajectories. The latter effect gives rise to an infinite eddy diffusivity, i.e., a regime of superdiffusion [50].

\section{Limit of vanishing Stokes number}

Another noteworthy case is when the Stokes time $\tau$ is much smaller than the typical flow timescale $\tau_{F}$ (i.e., St $\ll 1$, with St the Stokes number $\tau / \tau_{F}$ ) but $\gamma \boldsymbol{B} \tau$ is independent of $\tau$. By introducing the dimensionless magnetic field $\boldsymbol{B}^{*}=\gamma \tau \boldsymbol{B}$, Eq. (27) becomes

$$
\mathbb{A} \hat{\boldsymbol{v}}(z)=\hat{\boldsymbol{u}}(z)+\sqrt{2 D_{0}} \hat{\boldsymbol{\eta}}(z)
$$

with

$$
\mathbb{A}^{\mu \nu}=\delta^{\mu \nu}+B^{* i} \epsilon^{\mu \sigma \nu}
$$

Upon inverting the Laplace transform, the equation for the particle velocity is

$$
\frac{d \xi}{d t}(t)=\mathbb{A}^{-1} \boldsymbol{u}(\boldsymbol{\xi}(t), t)+\sqrt{2 D_{0}} \mathbb{A}^{-1} \boldsymbol{\eta}(t)
$$

The system is equivalent to a tracer advected by a compressible drift field $\tilde{\boldsymbol{u}}=\mathbb{A}^{-1} \boldsymbol{u}$ and subject to an anisotropic diffusion coefficient $\tilde{\sigma}=\sqrt{2 D_{0}} \mathbb{A}^{-1}$. The eddy diffusivity is in this case

$$
\mathbb{D}=D_{0} \mathbb{A}^{-1}\left(\mathbb{A}^{-1}\right)^{T}+\mathbb{A}^{-1} \operatorname{Sym}\left(\hat{\mathbb{C}}_{00}(0)\right)\left(\mathbb{A}^{-1}\right)^{T} .
$$

The limit of $\boldsymbol{B}^{*} \rightarrow 0$ then recovers Taylor's formula for tracer particles. Notice that in Eq. (32) $\mathbb{A}^{\mu \nu}$ is by definition dimensionless. 


\section{Inclusion of the Coriolis force}

The inclusion of Coriolis force in the Basset-Boussinesq-Oseen model in the geostrophic approximation limit and neglecting the history-force term yields [51]

$$
\frac{d \boldsymbol{v}}{d t}(t)=\frac{\boldsymbol{u}(\boldsymbol{\xi}(t), t)-\boldsymbol{v}(t)}{\tau}+\beta \frac{d \boldsymbol{u}(\boldsymbol{\xi}(t), t)}{d t}+(1-\beta) \boldsymbol{g}-2 \boldsymbol{\Omega} \times[\boldsymbol{v}(t)-\beta \boldsymbol{u}(\boldsymbol{\xi}(t), t)]+\frac{\sqrt{2 D_{0}}}{\tau} \boldsymbol{\eta}(t)
$$

According to the geostrophic approximation, the centrifugal force is a small constant term which can be absorbed in a redefinition of $\boldsymbol{g}$. The Fourier-Laplace transform of (35) yields

$$
\hat{\mathbb{A}}^{\mu v}(z) \hat{v}^{v}(z)=\hat{\mathbb{B}}^{\mu \nu}(z) \hat{u}^{\nu}(z)+\frac{1-\beta}{\tau} g^{\mu}+\frac{\sqrt{2 D_{0}}}{\tau} \hat{\eta}^{\mu}(z),
$$

where we define

$$
\begin{aligned}
& \hat{\mathbb{A}}^{\mu \nu}(z)=\left(z+\frac{1}{\tau}\right) \delta^{\mu \nu}+2 \Omega^{\sigma} \epsilon^{\mu \sigma \nu}, \\
& \hat{\mathbb{B}}^{\mu \nu}(z)=\left(\beta z+\frac{1}{\tau}\right) \delta^{\mu \nu}+2 \beta \Omega^{\sigma} \epsilon^{\mu \sigma \nu} .
\end{aligned}
$$

In Eq. (35) and in other occasions below, we use the Einstein convention for repeated indexes labeling tensor spatial components. Generalized Taylor's formula becomes

$$
\mathbb{D}=\frac{D_{0}}{\tau^{2}} \hat{\mathbb{A}}^{-1}(0)\left(\hat{\mathbb{A}}^{-1}\right)^{T}(0)+\hat{\mathbb{A}}^{-1}(0) \hat{\mathbb{B}}(0) \operatorname{Sym}\left(\hat{\mathbb{C}}_{00}(0)\right) \hat{\mathbb{B}}^{T}(0)\left(\hat{\mathbb{A}}^{-1}\right)^{T}(0) .
$$

\section{Limit of vanishing carrier velocity field}

If we consider a situation of zero flow, then the diffusion is caused only by the molecular white noise. We, thus, recover (30) with $\gamma=2$ and $B=\|\Omega\|$.

\section{Limit of vanishing Stokes number at fixed Rossby number}

It is again worth considering the limit of small Stokes time $\tau$ with respect to the flow timescale, while holding fixed the Rossby number Ro $=1 /(\tau \Omega)$.

If we define the constant matrices

$$
\begin{aligned}
& \mathbb{A}^{\mu \nu}=\delta^{\mu \nu}+2 \tau \Omega^{\sigma} \epsilon^{\mu \sigma \nu}, \\
& \mathbb{B}^{\mu \nu}=\delta^{\mu \nu}+2 \beta \tau \Omega^{\sigma} \epsilon^{\mu \sigma \nu},
\end{aligned}
$$

we can write the equation for the particle velocity as

$$
\frac{d \boldsymbol{\xi}}{d t}(t)=\mathbb{A}^{-1} \mathbb{B} \boldsymbol{u}(\boldsymbol{\xi}(t), t)+\sqrt{2 D_{0}} \mathbb{A}^{-1} \boldsymbol{\eta}(t) .
$$

The same considerations apply here as for Eq. (33). The eddy diffusivity becomes

$$
\mathbb{D}=D_{0} \mathbb{A}^{-1}\left(\mathbb{A}^{-1}\right)^{T}+\mathbb{A}^{-1} \mathbb{B} \operatorname{Sym}\left(\hat{\mathbb{C}}_{00}(0)\right) \mathbb{B}^{T}\left(\mathbb{A}^{-1}\right)^{T} .
$$

In order to illustrate the relative importance of the distinct contributions to this formula, it is expedient to consider a simple three-dimensional model consisting of a shear flow on a rotating plane (see Fig. 1). The angular velocity $\boldsymbol{\Omega}$ is oriented along the third axis $\boldsymbol{e}_{3}$ and the randomly fluctuating shear flow is

$$
\boldsymbol{u}(\boldsymbol{x}, t)=u\left(x_{2}, x_{3}, t\right) \boldsymbol{e}_{1},
$$

with $\boldsymbol{e}_{1}$ being the unit vector along the first coordinate axis. 


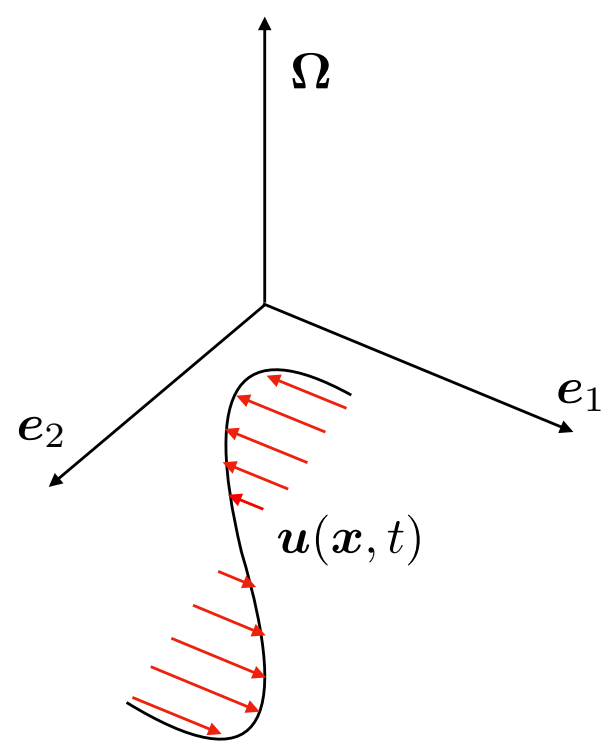

FIG. 1. Sketch of a shear flow along the direction $\boldsymbol{e}_{1}$ in a reference frame with angular velocity $\boldsymbol{\Omega}$ along $\boldsymbol{e}_{3}$.

Under these hypotheses, the tensor $\mathbb{C}_{00}(t)$ in Eq. (38) has only one nonvanishing component: $\mathbb{C}_{00}^{11}(t)=C(t)$. Thus, upon introducing the vector

$$
\boldsymbol{M}=\mathbb{A}^{-1} \mathrm{~B} \boldsymbol{e}_{x}=\frac{1}{1+4 / \mathrm{Ro}^{2}}\left(\begin{array}{c}
1+4 \beta / \mathrm{Ro}^{2} \\
2(\beta-1) / \mathrm{Ro} \\
0
\end{array}\right) .
$$

The generalized Taylor formula takes the form

$$
\mathrm{D}=\frac{D_{0}}{1+4 / \mathrm{Ro}^{2}} \mathbb{1}+\boldsymbol{M} \otimes \boldsymbol{M} \hat{C}(0),
$$

where

$$
\hat{C}(0)=\int_{0}^{\infty} d t C(t)
$$

In components, (40) is

$$
\mathrm{D}=\frac{1}{1+4 / \mathrm{Ro}^{2}}\left[\begin{array}{ccc}
D_{0}+\frac{\left(1+4 \beta / \mathrm{Ro}^{2}\right)^{2}}{1+4 / \mathrm{Ro}^{2}} \hat{C}(0) & \frac{2\left(1+4 \beta / \mathrm{Ro}^{2}\right)(\beta-1) / \mathrm{Ro}}{1+4 / \mathrm{Ro}^{2}} \hat{C}(0) & 0 \\
\frac{2\left(1+4 \beta / \mathrm{Ro}^{2}\right)(\beta-1) / \mathrm{Ro}}{1+4 / \mathrm{Ro}^{2}} \hat{C}(0) & D_{0}+\frac{4(\beta-1)^{2} / \mathrm{Ro}^{2}}{1+4 / \mathrm{Ro}^{2}} \hat{C}(0) & 0 \\
0 & 0 & D_{0}\left(1+4 / \mathrm{Ro}^{2}\right)
\end{array}\right] .
$$

It is instructive to analyze the behavior of the trace of the eddy diffusivity as a function of the Rossby number Ro:

$$
\operatorname{Tr} \mathrm{D}=D_{0}\left(1+\frac{2}{\left(1+4 / \mathrm{Ro}^{2}\right)}\right)+\frac{\left(1+4 \beta / \mathrm{Ro}^{2}\right)^{2}+4(\beta-1)^{2} / \mathrm{Ro}^{2}}{\left(1+4 / \mathrm{Ro}^{2}\right)^{2}} \hat{C}(0) .
$$

$\operatorname{Tr} \mathrm{D}$ is a monotonic function of Ro for fixed $\beta$ and $D_{0}$ :

$$
\frac{1}{\left(\beta^{2}-1\right) \hat{C}(0)-2 D_{0}} \frac{d}{d \operatorname{Ro}} \operatorname{Tr} \mathbb{D}=-\frac{8 \operatorname{Ro}}{\left(\operatorname{Ro}^{2}+4\right)^{2}} .
$$


In the limit of vanishing Rossby number (i.e., ideally an infinite value of $\Omega$ ), we get

$$
\lim _{\operatorname{Ro\downarrow } \downarrow} \operatorname{Tr} \mathbb{D}=D_{0}+\beta^{2} \hat{C}(0) .
$$

The opposite limit of large Rossby (i.e., the absence of rotation) recovers the expression of the tracer particle model

$$
\lim _{\operatorname{Ro} \uparrow \infty} \operatorname{Tr} \mathbb{D}=3 D_{0}+\hat{C}(0)
$$

For $\beta<1, \operatorname{Tr} \mathbb{D}$ always grows with respect to Ro. For light particles $(\beta>1)$, instead, the $\operatorname{Tr} \mathbb{D}$ may be monotonically decreasing or increasing depending upon whether $\hat{C}(0)$ is respectively higher or lower than the threshold value:

$$
\hat{C}_{\star}(0) \equiv \frac{2 D_{0}}{\beta^{2}-1}
$$

For incompressible carrier fields $\boldsymbol{u}, \hat{C}(0)$ is always positive. Hence, it is clear that only for $\beta>1$ a decreasing behavior is possible.

\section{MAXEY-RILEY MODEL}

We now turn to the extension of Taylor's formula to the now "canonical" Maxey-Riley model [29] inclusive of the time derivatives along fluid trajectories and the Faxén friction [30,31]:

$$
\begin{aligned}
\frac{d \boldsymbol{v}}{d t}(t)= & \frac{\boldsymbol{u}(\boldsymbol{\xi}(t), t)-\boldsymbol{v}(t)+\frac{1}{6} r_{p}^{2} \nabla^{2} \boldsymbol{u}(\boldsymbol{\xi}(t), t)}{\tau}+\beta \frac{\mathrm{D} \boldsymbol{u}}{\mathrm{D} t}(\boldsymbol{\xi}(t), t)+\frac{\beta}{30} r_{p}^{2} \frac{d}{d t} \nabla^{2} \boldsymbol{u}(\boldsymbol{\xi}(t), t) \\
& +\sqrt{\frac{3 \beta}{\pi \tau}} \int_{0}^{t} \frac{d s}{\sqrt{t-s}} \frac{d}{d s}\left[\boldsymbol{u}(\boldsymbol{\xi}(s), s)-\boldsymbol{v}(s)+\frac{1}{6} r_{p}^{2} \nabla^{2} \boldsymbol{u}(\boldsymbol{\xi}(s), s)\right]+\frac{\sqrt{2 D_{0}}}{\tau} \boldsymbol{\eta}(t)
\end{aligned}
$$

The convective derivative evaluated along the inertial particle trajectory

$$
\frac{\mathrm{D} \boldsymbol{u}}{\mathrm{D} t}(\boldsymbol{\xi}(t), t)=\left[\partial_{t} \boldsymbol{u}(\boldsymbol{x}, t)+\boldsymbol{u}(\boldsymbol{x}, t) \cdot \nabla \boldsymbol{u}(\boldsymbol{x}, t)\right]_{\boldsymbol{x}=\boldsymbol{\xi}(t)}
$$

represents a higher order correction with respect to the Basset-Boussinesq-Oseen equation in the Stokes number, which still needs to be small [29]. The Faxén drag force accounts for higher order corrections in particles size [52] by including $O\left(r_{p}^{2} / L^{2}\right)$ corrections to the Stokes drag. These higher order corrections with respect to Stokes number and particle radius are often needed in applications $[11,42]$. For the sake of simplicity, we do not discuss here external forces. Upon removing the initial transient, taking the Fourier-Laplace transform, and recalling (18), we get

$$
\begin{aligned}
\hat{\boldsymbol{v}}(z)= & \frac{1}{a(z)}\left\{\beta \frac{\widehat{\mathrm{D} \boldsymbol{u}}}{\mathrm{D} t}(z)+\left[a(z)-z-\frac{1}{\tau}\right]\left[\hat{\boldsymbol{u}}(z)+\frac{1}{6} r_{p}^{2} \widehat{\nabla^{2} \boldsymbol{u}}(z)\right]\right\} \\
& +\frac{1}{\tau a(z)}\left[\hat{\boldsymbol{u}}(z)+\frac{1}{6} r_{p}^{2} \widehat{\nabla^{2} \boldsymbol{u}}(z)+\sqrt{2 D_{0}} \hat{\boldsymbol{\eta}}(z)+z \frac{\beta}{30} r_{p}^{2} \widehat{\nabla^{2} \boldsymbol{u}}(z)\right] .
\end{aligned}
$$

Again, the model can be couched into the form (4b) with all tensors $\mathbb{K}_{i}$ 's having the form of the identity matrix times scalar functions $K_{i} i=0, \ldots, 3$. By comparing to Eqs. (19), we 
see that

$$
\begin{aligned}
& \hat{K}_{0}(z)=1-\frac{z}{a(z)}, \quad \hat{K}_{1}(z)=\frac{1}{a(z)}, \quad \hat{\boldsymbol{f}}_{1}(z)=\beta \frac{\widehat{\mathrm{Du}}}{\mathrm{D} t}(z)+\frac{\sqrt{2 D_{0}}}{\tau} \hat{\boldsymbol{\eta}}(z), \\
& \hat{K}_{2}(z)=\tau \hat{K}_{0}(z), \quad \hat{\boldsymbol{f}}_{2}(z)=\frac{1}{6 \tau} r_{p}^{2} \widehat{\nabla^{2} \boldsymbol{u}}(z), \quad \hat{K}_{3}(z)=\frac{\tau z}{a(z)}, \quad \hat{\boldsymbol{f}}_{3}(z)=\frac{\beta}{30 \tau} r_{p}^{2} \widehat{\nabla^{2} \boldsymbol{u}}(z) .
\end{aligned}
$$

The scalar kernels satisfy

$$
\begin{aligned}
& \hat{K}_{0}(0)=\int_{0}^{\infty} d t K_{0}(t)=1, \\
& \hat{K}_{i}(0)=\int_{0}^{\infty} d t K_{i}(t)=\tau, \quad i=1,2,
\end{aligned}
$$

while $\hat{K}_{3}(0)=0$. This latter fact implies that $\boldsymbol{f}_{3}$ does not give any contribution to the eddy diffusivity. Upon applying the general result (14), we get

$$
\begin{aligned}
\mathrm{D}= & D_{0} \mathbb{1}+\operatorname{Sym} \int_{t_{0}}^{\infty} d t\left\langle\left[\delta \boldsymbol{u}(\boldsymbol{\xi}(t), t)+\beta \tau \delta \frac{\mathrm{D} \boldsymbol{u}}{\mathrm{D} t}(\boldsymbol{\xi}(t), t)+\frac{1}{6} r_{p}^{2} \delta \nabla^{2} \boldsymbol{u}(\boldsymbol{\xi}(t), t)\right]\right. \\
& \left.\otimes\left[\delta \boldsymbol{u}\left(\boldsymbol{\xi}\left(t_{0}\right), t_{0}\right)+\beta \tau \delta \frac{\mathrm{D} \boldsymbol{u}}{\mathrm{D} t}\left(\boldsymbol{\xi}\left(t_{0}\right), t_{0}\right)+\frac{1}{6} r_{p}^{2} \delta \nabla^{2} \boldsymbol{u}\left(\boldsymbol{\xi}\left(t_{0}\right), t_{0}\right)\right]\right\rangle,
\end{aligned}
$$

where $t_{0}$ denotes the typical time it takes before correlation functions attain a stationary form, and

$$
\begin{aligned}
\delta \boldsymbol{u}(\boldsymbol{\xi}(t), t) & =\boldsymbol{u}(\boldsymbol{\xi}(t), t)-\langle\boldsymbol{u}(\boldsymbol{\xi}(t), t)\rangle, \\
\delta \frac{\mathrm{D} \boldsymbol{u}}{\mathrm{D} t}(\boldsymbol{\xi}(t), t) & =\frac{\mathrm{D} \boldsymbol{u}}{\mathrm{D} t}(\boldsymbol{\xi}(t), t)-\left\langle\frac{\mathrm{D} \boldsymbol{u}}{\mathrm{D} t}(\boldsymbol{\xi}(t), t)\right\rangle, \\
\delta \nabla^{2} \boldsymbol{u}(\boldsymbol{\xi}(t), t) & =\nabla^{2} \boldsymbol{u}(\boldsymbol{\xi}(t), t)-\left\langle\nabla^{2} \boldsymbol{u}(\boldsymbol{\xi}(t), t)\right\rangle .
\end{aligned}
$$

By comparing Eqs. (44) and (26), we clearly see the Maxey-Riley and Basset-Boussinesq-Oseen models tend to coincide when $r_{p} / L$ and $\beta \tau / \tau_{F}$ are $\ll 1$, with $\tau_{F}$ and $L$ being the characteristic timescale and length scale of the flow, respectively.

Equation (44) generalizes results previously given in literature (see, e.g., Ref. [53]), where explicit expressions for the eddy diffusivity had been derived in the case of heavy particles [i.e., $\beta=0$ when only the Stokes drag in Eq. (41) survives].

\section{MODELS INCLUDING LIFT FORCES}

Further higher order corrections due to particle size and higher Stokes numbers include lift forces. The earliest model was introduced by Saffman in 1965 [54,55] for small solid particles in shear flows. An adapted form of this model is widely applied to describe dynamics in three-dimensional flows [56]. A lot of different empirical models have been proposed since then, taking into account different sizes and shapes of particles, wall effects, momentum transfer between the carrier fluid and the inner fluid inside the particle-which is meaningful if that particle is a bubble-or finite Reynolds numbers [57-61]. The typically surmised form of the lift force acting on a spherical particle is

$$
\boldsymbol{F}_{L}=C_{L} \rho_{f} \frac{4}{3} \pi r_{p}^{3}[\boldsymbol{v}(t)-\boldsymbol{u}(\boldsymbol{\xi}(t), t)] \times \omega(\boldsymbol{\xi}(t), t),
$$

where $\boldsymbol{\omega}=\boldsymbol{\nabla} \times \boldsymbol{u}$ is the vorticity and $C_{L}$ is the lift coefficient, which in general can be solely determined by fitting experimental data. 
It is not in the scope of this article to provide a general view over lift force models, which is a vast phenomenology as said above. We want instead to provide an example about how to obtain an expression of the eddy diffusivity via generalized Taylor's formula. To do so, we focus on the Saffman model, for which [56]

$$
C_{L}=\frac{6.46}{\frac{4}{3} \pi r_{p}} \sqrt{\frac{v}{\|\omega(\xi(t), t)\|}}
$$

The equation of motion is

$$
\begin{aligned}
\frac{d \boldsymbol{v}}{d t}(t)= & \frac{\boldsymbol{u}(\boldsymbol{\xi}(t), t)-\boldsymbol{v}(t)+\frac{1}{6} r_{p}^{2} \nabla^{2} \boldsymbol{u}(\boldsymbol{\xi}(t), t)}{\tau}+\beta \frac{\mathrm{D} \boldsymbol{u}(\boldsymbol{\xi}(t), t)}{\mathrm{D} t}+\frac{\beta}{30} r_{p}^{2} \frac{d}{d t} \nabla^{2} \boldsymbol{u}(\boldsymbol{\xi}(t), t) \\
& +\sqrt{\frac{3 \beta}{\pi \tau}} \int_{0}^{t} \frac{d s}{\sqrt{t-s}} \frac{d}{d s}\left[\boldsymbol{u}(\boldsymbol{\xi}(s), s)-\boldsymbol{v}(s)+\frac{1}{6} r_{p}^{2} \nabla^{2} \boldsymbol{u}(\xi(s), s)\right] \\
& +\frac{6.46 \beta}{2 \pi r_{p}} \sqrt{\frac{v}{\|\omega(\boldsymbol{\xi}(t), t)\|}}[\boldsymbol{v}(t)-\boldsymbol{u}(\boldsymbol{\xi}(t), t)] \times \boldsymbol{\omega}(\boldsymbol{\xi}(t), t)+\frac{\sqrt{2 D_{0}}}{\tau} \boldsymbol{\eta}(t) .
\end{aligned}
$$

The vorticity sets the typical timescale for advection, i.e., $\tau_{F}=\max (1 /\|\omega\|)$. Hence, upon recalling that $\tau=3 r_{p}^{2} /(\nu \beta)$, the ratio between the Saffman force per unit mass and the Stokes drag is

$$
\frac{\frac{2}{3} \beta \frac{6.46}{4 / 3 \pi r_{p}} \sqrt{\frac{v}{\|\omega\|}}\|[\boldsymbol{v}-\boldsymbol{u}] \times \boldsymbol{\omega}\|}{\|\boldsymbol{u}-\boldsymbol{v}\| / \tau} \leqslant \frac{2}{3} \beta \frac{6.46}{4 / 3 \pi} \sqrt{\frac{v}{\|\omega\| r_{p}^{2}}} \tau\|\omega\| \sim O(\sqrt{\mathrm{St}}) .
$$

As a result, Saffman's lift force is always negligible at sufficiently low Stokes times, or whenever $\rho_{p} \gg \rho_{f}$, that is $\beta \ll 1$. Saffman's model holds true, if $\operatorname{Re}_{p} \sim 0$ and

$$
\max \frac{\left\|\boldsymbol{\Omega}_{p}\right\| r_{p}^{2}}{\|\boldsymbol{v}-\boldsymbol{u}\|} \ll 1 \quad \text { and } \quad \max \frac{\sqrt{\|\boldsymbol{\omega}\| / v}}{\|\boldsymbol{v}-\boldsymbol{u}\| / v} \gg 1,
$$

with $\boldsymbol{\Omega}_{p}$ being the particle angular velocity.

In addition to the force terms of the Maxey-Riley model (43), there now appear two extra terms:

$$
\hat{K}_{4}(z)=\hat{K}_{1}(z) \text { and } \hat{\boldsymbol{f}}_{4}(z)=\hat{\boldsymbol{f}}_{L}(z)
$$

where $\hat{f}_{L}$ is the time Laplace transform of the lift force:

$$
f_{L}(\xi(t), t)=\frac{6.46 \beta}{2 \pi r_{p}} \sqrt{\frac{v}{\|\omega(\xi(t), t)\|}}[v(t)-\boldsymbol{u}(\xi(t), t)] \times \omega(\xi(t), t) .
$$

A straightforward application of generalized Taylor's formula (14) yields

$$
\begin{aligned}
\mathbb{D}= & D_{0} \mathbb{1}+\operatorname{Sym} \int_{t_{0}}^{\infty} d t\left\langle\left[\delta \boldsymbol{u}(\boldsymbol{\xi}(t), t)+\beta \tau \delta \frac{\mathrm{D} \boldsymbol{u}}{\mathrm{D} t}(\boldsymbol{\xi}(t), t)+\frac{1}{6} r_{p}^{2} \delta \nabla^{2} \boldsymbol{u}(\boldsymbol{\xi}(t), t)+\tau \delta \boldsymbol{f}_{L}(\boldsymbol{\xi}(t), t)\right]\right. \\
& \left.\otimes\left[\delta \boldsymbol{u}\left(\boldsymbol{\xi}\left(t_{0}\right), t_{0}\right)+\beta \tau \delta \frac{\mathrm{D} \boldsymbol{u}}{\mathrm{D} t}\left(\boldsymbol{\xi}\left(t_{0}\right), t_{0}\right)+\frac{1}{6} r_{p}^{2} \delta \nabla^{2} \boldsymbol{u}\left(\boldsymbol{\xi}\left(t_{0}\right), t_{0}\right)+\tau \delta \boldsymbol{f}_{L}\left(\boldsymbol{\xi}\left(t_{0}\right), t_{0}\right)\right]\right\rangle .
\end{aligned}
$$

Equation (52) opens the way for assessing the contribution of the autocorrelation of the lift force and its cross correlations with the other terms to the eddy diffusivity. This can be done, in way of example, by analyzing trajectories from available radar data or numerical simulations.

It should be noted that Eqs. (48) and (52) do not contain lift terms depending on the angular velocity $\boldsymbol{\Omega}_{p}$ of the particle, the so-called Magnus effect. Indeed, among higher order corrections (see Eqs. (2.17)-(4.15) in Ref. [54] and Eq. (4) in Ref. [56]), a lift force acting on the particle of the 
form [62]

$$
\rho_{f} r_{p}^{3} \pi \boldsymbol{\Omega}_{p} \times[\boldsymbol{v}(t)-\boldsymbol{u}(\boldsymbol{\xi}(t), t)]
$$

should be added to Eq. (46). However, the ratio between this term per unit mass and the Stokes drag is

$$
\frac{\frac{2}{3} \beta \pi r_{p}^{3}\left\|\boldsymbol{\Omega}_{p} \times[\boldsymbol{v}(t)-\boldsymbol{u}(\boldsymbol{\xi}(t), t)]\right\|}{\frac{4}{3} \pi r_{p}^{3}\|\boldsymbol{v}(t)-\boldsymbol{u}(\boldsymbol{\xi}(t), t)\| / \tau} \leqslant \beta\left\|\boldsymbol{\Omega}_{p}\right\| \tau \leqslant \beta \frac{\left\|\boldsymbol{\Omega}_{p}\right\|}{\|\boldsymbol{\omega}\|} \mathrm{St} .
$$

This ratio is of order $O(\mathrm{St})$, while the ratio between Saffman lift and Stokes drag is $O(\sqrt{\mathrm{St}})$. This justifies why the Magnus term [62] is often neglected for small solid particles, unless the angular velocity is high. We do not take into explicit account this case. The reason is that this latter case can be accounted for in Eq. (52) by a redefinition of $\boldsymbol{f}_{L}$. For a freely rotating sphere the difference is small though, since $\boldsymbol{\Omega}_{p}=1 / 2 \boldsymbol{\omega}$ [54].

\section{CONCLUSIONS}

We analyzed general conditions under which a generalized Taylor eddy-diffusivity formula applies to inertial particle models.

It is worth emphasizing that Taylor's formula for the Basset-Boussinesq-Oseen model of inertial particle dynamics with the inclusion of the Brownian force is formally the same as the Taylor's formula for tracer particles. The equivalence is, however, only formal. Since the time integral of the fluid velocity autocorrelation function is carried out along particle trajectories, the well-known mismatch between fluid and particle trajectories leads in general to different eddy diffusivities.

In the case of the Maxey-Riley model, new terms appear in the expression for the eddy diffusivity with respect to the tracer case and thus with respect to the Basset-Boussinesq-Oseen model. We also discussed under which conditions the two models admit the same formal expression for the eddy diffusivity. Similar results hold as well for lift forces.

Our analysis encompasses, as special cases of interest in applications, the two relevant examples of particle dynamics forced by the Coriolis contribution (for application to dispersions in geophysical flows) and the Lorentz force (for application to dispersions of charged particles in electrically neutral flows). In this latter case, we proved that in the limit of small inertia (i.e., St $\downarrow 0$ ) and magnetic field $\boldsymbol{B}^{*}$ such that $\left\|\boldsymbol{B}^{*}\right\|$ is independent of St, the inertial particle dynamics reduces to a tracer dynamics with a carrier flow which now becomes compressible. Clustering phenomena induced by the magnetic field are thus expected to emerge. For a vanishing carrier flow, the combined roles of Brownian motion and magnetic field has been proved to give rise to a smaller eddy diffusivity than the molecular diffusivity $D_{0}$. Transport depletion is thus expected in applications involving the magnetic field. Similar conclusions can be obtained for the Coriolis contribution. The mathematical structure of this term is indeed very similar to the Lorentz force. To summarize the several eddy-diffusivity expressions, we collected all of them in Table I.

Taylor's formula for tracer dispersion has ubiquitous applications in the study of turbulent transport. We thus expect that our analysis will be useful for further investigations of large-scale transport properties of inertial particles under the action of different forcing mechanisms.

\section{ACKNOWLEDGMENTS}

The research of S. Boi is funded by the AtMath Collaboration at the University of Helsinki. P. Muratore-Ginanneschi also acknowledges support from the AtMath Collaboration. Useful discussions with G. Falkovich and R. Ferrari are warmly acknowledged. 
TABLE I. Generalized Taylor formulas.

Model

Eddy diffusivity

Basset-Boussinesq-Oseen equation $\lim _{t \uparrow \infty} \operatorname{Sym} \int_{0}^{t} d s\langle\delta \boldsymbol{u}(\boldsymbol{\xi}(s), s) \otimes \delta \boldsymbol{u}(\boldsymbol{\xi}(t), t)\rangle$

plus white noise and constant gravity

Basset-Boussinesq-Oseen equation plus white noise and Lorentz force

$$
\begin{gathered}
\frac{D_{0}}{\tau^{2}} \hat{\mathbb{A}}^{-1}(0)\left(\hat{\mathbb{A}}^{-1}\right)^{T}(0)+\frac{1}{\tau^{2}} \hat{\mathbb{A}}^{-1}(0) \operatorname{Sym}\left(\hat{\mathbb{C}}_{00}(0)\right)\left(\hat{\mathbb{A}}^{-1}\right)^{T}(0) \\
\text { where } \\
\left(\hat{\mathbb{A}}^{-1}\right)^{\mu \nu}(z)=\frac{1}{a^{2}(z)+\gamma^{2}\|\boldsymbol{B}\|^{2}}\left[a(z) \delta^{\mu \nu}-\gamma B^{i} \epsilon^{\mu \sigma v}+\frac{\gamma^{2}}{a(z)} B^{k} B^{j}\right] \\
a(z)=z+\frac{1}{\tau}+\sqrt{\frac{3 \beta z}{\tau}} \\
\gamma=\frac{q}{\frac{4}{3} \pi r_{p}^{3} \rho_{p}}
\end{gathered}
$$

Basset-Boussinesq-Oseen equation plus white noise and Lorentz force $\operatorname{diag}\left(\frac{D_{0}}{1+\gamma^{2} B^{2} \tau^{2}}, \frac{D_{0}}{1+\gamma^{2} B^{2} \tau^{2}}, D_{0}\right)$ (flow at rest, constant magnetic field $\boldsymbol{B}=(0,0, B))$

Basset-Boussinesq-Oseen equation plus white noise and Lorentz force (limit of vanishing Stokes number and $\boldsymbol{B}^{*}=\gamma \tau \boldsymbol{B}$ )

Basset-Boussinesq-Oseen equation plus constant gravity, white noise and Coriolis force,

Basset force neglected

$$
\begin{gathered}
D_{0} \mathbb{A}^{-1}\left(\mathbb{A}^{-1}\right)^{T}+\mathbb{A}^{-1} \operatorname{Sym}\left(\hat{\mathbb{C}}_{00}(0)\right)\left(\mathbb{A}^{-1}\right)^{T} \\
\text { where } \\
\mathbb{A}^{\mu \nu}=\delta^{\mu \nu}+B^{* i} \epsilon^{\mu \sigma v}
\end{gathered}
$$

$$
\begin{gathered}
\frac{D_{0}}{\tau^{2}} \hat{\mathbb{A}}^{-1}(0)\left(\hat{\mathbb{A}}^{-1}\right)^{T}(0)+\hat{\mathbb{A}}^{-1}(0) \hat{\mathrm{B}}(0) \\
\operatorname{Sym}\left(\hat{\mathbb{C}}_{00}(0)\right) \hat{\mathbb{B}}^{T}(0)\left(\hat{\mathbb{A}}^{-1}\right)^{T}(0)
\end{gathered}
$$

where

$\hat{\mathrm{A}}^{\mu \nu}(z)=\left(z+\frac{1}{\tau}\right) \delta^{\mu \nu}+2 \Omega^{\sigma} \epsilon^{\mu \sigma v}$

$\hat{\mathbb{B}}^{\mu \nu}(z)=\left(\beta z+\frac{1}{\tau}\right) \delta^{\mu \nu}+2 \beta \Omega^{\sigma} \epsilon^{\mu \sigma \nu}$

Basset-Boussinesq-Oseen equation plus white noise, constant gravity and Coriolis force (flow at rest, constant angular

\begin{tabular}{|c|c|}
\hline $\begin{array}{l}\text { Maxey-Riley equation } \\
\text { (including white noise, Faxén, } \\
\text { and Auton terms) }\end{array}$ & $\begin{array}{c}D_{0} \mathbb{1}+\operatorname{Sym} \int_{t_{0}}^{\infty} d t \\
\left\langle\left[\delta \boldsymbol{u}(\boldsymbol{\xi}(t), t)+\beta \tau \delta \frac{\mathrm{D} \boldsymbol{u}}{\mathrm{D} t}(\boldsymbol{\xi}(t), t)+\frac{1}{6} r_{p}^{2} \delta \nabla^{2} \boldsymbol{u}(\boldsymbol{\xi}(t), t)\right]\right. \\
\left.\otimes\left[\delta \boldsymbol{u}\left(\boldsymbol{\xi}\left(t_{0}\right), t_{0}\right)+\beta \tau \delta \frac{\mathrm{D} \boldsymbol{u}}{\mathrm{D} t}\left(\boldsymbol{\xi}\left(t_{0}\right), t_{0}\right)+\frac{1}{6} r_{p}^{2} \delta \nabla^{2} \boldsymbol{u}\left(\boldsymbol{\xi}\left(t_{0}\right), t_{0}\right)\right]\right\rangle\end{array}$ \\
\hline Maxey-Riley equation + lift force & $\begin{array}{l}\mathrm{D}=D_{0} \mathbb{1}+\operatorname{Sym} \int_{t_{0}}^{\infty} d t \\
\left\langle\left[\delta \boldsymbol{u}(\boldsymbol{\xi}(t), t)+\beta \tau \delta \frac{\mathrm{D} \boldsymbol{u}}{\mathrm{D} t}(\boldsymbol{\xi}(t), t)\right.\right. \\
\left.+\frac{1}{6} r_{p}^{2} \delta \nabla^{2} \boldsymbol{u}(\boldsymbol{\xi}(t), t)+\tau \delta \boldsymbol{f}_{L}(\boldsymbol{\xi}(t), t)\right] \\
\otimes\left[\delta \boldsymbol{u}\left(\boldsymbol{\xi}\left(t_{0}\right), t_{0}\right)+\beta \tau \delta \frac{\mathrm{D} \boldsymbol{\mathrm { D }}}{\mathrm{D} t}\left(\boldsymbol{\xi}\left(t_{0}\right), t_{0}\right)\right. \\
\left.\left.+\frac{1}{6} r_{p}^{2} \delta \nabla^{2} \boldsymbol{u}\left(\boldsymbol{\xi}\left(t_{0}\right), t_{0}\right)+\tau \delta \boldsymbol{f}_{L}\left(\boldsymbol{\xi}\left(t_{0}\right), t_{0}\right)\right]\right\rangle\end{array}$ \\
\hline
\end{tabular}
velocity $\boldsymbol{\Omega}=(0,0, \Omega))$

Basset-Boussinesq-Oseen equation plus white noise, constant gravity and Coriolis force,

Basset force neglected.

$$
\operatorname{diag}\left(\frac{D_{0}}{1+4 \Omega^{2} \tau^{2}}, \frac{D_{0}}{1+4 \Omega^{2} \tau^{2}}, D_{0}\right)
$$

(shear flow $\boldsymbol{u}(\boldsymbol{x}, t)=u\left(x_{2}, x_{3}, t\right) \boldsymbol{e}_{1}$, constant angular velocity $\boldsymbol{\Omega}=(0,0, \Omega)$, fixed Rossby number Ro $=1 /(\tau \Omega)$ ) 


\section{APPENDIX}

Proposition 1. Under Hypotheses I-III, generalized Taylor's identity (14) holds true for any dynamical model of the form (4).

To prove the claim, we need first to couch (10) into the form (11) which is more adapted to discuss the large time limit. This is done by first applying to (10) the double integral inversion formula over a triangular domain

$$
\operatorname{Sym} \int_{0}^{t} d s\langle\delta \boldsymbol{v}(t) \otimes \delta \boldsymbol{v}(s)\rangle=\sum_{i j=0}^{N} \operatorname{Sym} \int_{0}^{t} d s_{3} \int_{0}^{t} d s_{2} \int_{s_{2}}^{t} d s_{1} \mathbb{K}_{i}\left(t-s_{3}\right) \tilde{\mathbb{C}}_{i j}\left(s_{3}, s_{2}\right) \mathbb{K}_{j}^{T}\left(s_{1}-s_{2}\right) .
$$

Performing the sequence with the change of variables $s_{1}=u_{1}+s_{2}, s_{2}=t-u_{2}$, and $s_{3}=t-u_{3}$ yields (11), which, for reading convenience, we rewrite here as

$$
\begin{aligned}
& \operatorname{Sym} \int_{0}^{t} d s\langle\delta \boldsymbol{v}(t) \otimes \delta \boldsymbol{v}(s)\rangle \\
& =\sum_{i j=0}^{N} \operatorname{Sym} \int_{0}^{t} d u_{3} \int_{0}^{t} d u_{2} \int_{0}^{u_{2}} d u_{1} \mathbb{K}_{i}\left(u_{3}\right) \tilde{\mathbb{C}}_{i j}\left(t-u_{3}, t-u_{2}\right) \mathbb{K}_{j}^{T}\left(u_{1}\right) .
\end{aligned}
$$

We now invoke Hypotheses I and II. They ensure that (A2) [or equivalently (11)] is absolutely integrable in the large time limit. Before proving this claim, it is convenient to proceed to analyze its implications. Namely, if we take the limit under the integral and invoke Hypothesis III, upon applying once again the double integral inversion formula over a triangular domain, we obtain

$$
\mathbb{D}=\lim _{t \uparrow \infty} \operatorname{Sym} \int_{0}^{t} d s\langle\delta \boldsymbol{v}(t) \otimes \delta \boldsymbol{v}(s)\rangle=\sum_{i j=0}^{N} \operatorname{Sym} \int_{0}^{\infty} d u_{3} \int_{0}^{\infty} d u_{1} \mathbb{K}_{i}\left(u_{3}\right) \mathbb{F}_{i j}\left(u_{3}, u_{1}\right) \mathbb{K}_{j}^{T}\left(u_{1}\right),
$$

where by (13)

$$
\mathbb{F}_{i j}\left(u_{3}, u_{1}\right)=\int_{u_{1}}^{\infty} d u_{2} \begin{cases}\mathbb{C}_{i j}\left(u_{2}-u_{3}\right) & \forall u_{2} \geqslant u_{3} \\ \mathbb{C}_{j i}^{T}\left(u_{3}-u_{2}\right) & \forall u_{2}<u_{3}\end{cases}
$$

The kernel (A4) is in fact a function of $u_{1}-u_{3}$ alone and admits important simplifications. Namely, we notice that for $u_{1} \geqslant u_{3}$

$$
\mathbb{F}_{i j}\left(u_{3}, u_{1}\right)=\int_{0}^{\infty} d u \mathbb{C}_{i j}(u)-\int_{0}^{u_{1}-u_{3}} d u \mathbb{C}_{i j}(u),
$$

while for $u_{3}>u_{1}$ we find

$$
\mathbb{F}_{i j}\left(u_{3}, u_{1}\right)=\int_{0}^{\infty} d u \mathbb{C}_{i j}(u)+\int_{0}^{u_{3}-u_{1}} d u \mathbb{C}_{j i}^{T}(u) .
$$

Upon gleaning these observations, we conclude after a further application of (13) that

$$
\mathbb{F}_{i j}\left(u_{3}, u_{1}\right)=\int_{0}^{\infty} d u \mathbb{C}_{i j}(u)-\int_{0}^{u_{1}-u_{3}} d u \mathbb{C}_{i j}(u) \equiv \hat{\mathbb{C}}_{i j}(0)-\tilde{\mathbb{F}}_{i j}\left(u_{1}-u_{3}\right),
$$

where furthermore

$$
\tilde{\mathbb{F}}_{i j}(-t)=\int_{0}^{-t} d u \mathbb{C}_{i j}(u)=-\int_{0}^{t} d u \mathbb{C}_{i j}(-u)=-\int_{0}^{t} d u \mathbb{C}_{j i}^{T}(u)=-\tilde{\mathbb{F}}_{j i}^{T}(t) .
$$


We have now forged all the tools needed to prove the proposition. If we take the Sym operation under the integral sign and rename dummy integration and summation variables, we get

$$
\mathbb{D}=\sum_{i j=0}^{N} \int_{0}^{\infty} d u_{3} \int_{0}^{\infty} d u_{1} \mathbb{K}_{i}\left(u_{3}\right) \frac{\mathbb{F}_{i j}\left(u_{3}, u_{1}\right)+\mathbb{F}_{j i}^{T}\left(u_{1}, u_{3}\right)}{2} \mathbb{K}_{j}^{T}\left(u_{1}\right) .
$$

In view of (A5) and (A6), the chain of identities

$$
\begin{aligned}
\frac{\mathbb{F}_{i j}\left(u_{3}, u_{1}\right)+\mathbb{F}_{j i}^{T}\left(u_{1}, u_{3}\right)}{2} & =\frac{\hat{\mathbb{C}}_{i j}(0)+\hat{\mathbb{C}}_{j i}^{T}(0)}{2}-\frac{\tilde{\mathbb{F}}_{i j}\left(u_{1}-u_{3}\right)+\tilde{\mathbb{F}}_{j i}^{T}\left(u_{3}-u_{1}\right)}{2} \\
& =\frac{\hat{\mathbb{C}}_{i j}(0)+\hat{\mathbb{C}}_{j i}^{T}(0)}{2}-\frac{\tilde{\mathbb{F}}_{i j}\left(u_{1}-u_{3}\right)-\tilde{\mathbb{F}}_{i j}\left(u_{1}-u_{3}\right)}{2} \\
& =\frac{\hat{\mathbb{C}}_{i j}(0)+\hat{\mathbb{C}}_{j i}^{T}(0)}{2}
\end{aligned}
$$

holds true. Hence, the kernel in Eq. (A7) is independent of the integration variables $u_{1}, u_{3}$ and the double integral factorizes in the product of two integrals. As a consequence, (A7) reduces to generalized Taylor's formula (14), as claimed.

Finally, we can return to the proof that Hypotheses I and II are sufficient to guarantee that it is safe to apply the dominated convergence theorem to (A2). Namely, the chain of inequalities

$$
\begin{aligned}
\left|\int_{0}^{t} d s\left\langle\delta v^{m}(t) \delta v^{n}(s)\right\rangle\right| & \leqslant \sum_{i j=0}^{N} \int_{0}^{t} d s_{3} \int_{0}^{t} d s_{1} \int_{0}^{s_{1}} d s_{2}\left|\mathbb{K}_{i}^{m l}\left(s_{3}\right)\right|\left|\mathbb{K}_{j}^{n k}\left(s_{1}\right)\right|\left|\tilde{\mathbb{C}}_{i j}^{l k}\left(s_{3}, s_{2}\right)\right| \\
& \leqslant \int_{0}^{t} d s_{3} \int_{0}^{t} d s_{1} V^{m}\left(s_{3}\right) V^{n}\left(s_{1}\right) \int_{0}^{s_{1}} d s_{2} F\left(s_{3}-s_{2}\right)
\end{aligned}
$$

holds for

$$
V^{n}(t)=\sum_{i=0}^{N} \sum_{l=1}^{d}\left|\mathbb{K}_{i}^{n l}(t)\right|
$$

The innermost integral in Eq. (A9) satisfies

$$
0 \leqslant \int_{0}^{s_{1}} d s_{2} F\left(s_{3}-s_{2}\right)<\int_{-\infty}^{\infty} d s F(s) \equiv 2 F_{\star}
$$

since $F$ is positive, even, and integrable by hypothesis. We conclude that

$$
\lim _{t \uparrow \infty}\left|\int_{0}^{t} d s\left\langle\delta v^{m}(t) \delta v^{n}(s)\right\rangle\right|<2\left[(N+1) K_{\star} d\right]^{2} F_{\star}<\infty,
$$

with $K_{*}$ being defined in Eq. (5).

[1] G. I. Taylor, Diffusion by continuous movements, Proc. London Math. Soc. s2-20, 196 (1922).

[2] L. Biferale, A. Crisanti, M. Vergassola, and A Vulpiani, Eddy diffusivities in scalar transport, Phys. Fluids 7, 2725 (1995).

[3] S. Boi, A. Mazzino, and G. Lacorata, Explicit expressions for eddy-diffusivity fields and effective largescale advection in turbulent transport, J. Fluid Mech. 795, 524 (2016). 
[4] M. Cencini, A. Mazzino, S. Musacchio, and A. Vulpiani, Large-scale effects on meso-scale modeling for scalar transport, Phys. D (Amsterdam, Neth.) 220, 146 (2006).

[5] U. Frisch, Lecture on turbulence and lattice gas hydrodynamics, in Lecture Notes, NCAR-GTP Summer School June 1987, edited by J. R. Herring and J. C. McWilliams (World Scientific, Singapore, 1987), pp. 219-371.

[6] A. Mazzino, Effective correlation times in turbulent scalar transport, Phys. Rev. E 56, 5500 (1997).

[7] A. Mazzino, S. Musacchio, and A. Vulpiani, Multiple-scale analysis and renormalization for preasymptotic scalar transport, Phys. Rev. E 71, 011113 (2005).

[8] R. H. Kraichnan, Eddy viscosity and diffusivity: Exact formulas and approximations, Complex Syst. 1, 805 (1987).

[9] J. Majda and P. R. Kramer, Simplified models for turbulent diffusion: Theory, numerical modeling, and physical phenomena, Phys. Rep. 314, 237 (1999).

[10] P. K. Kundu, I. M. Cohen, and D. R. Dowling, Fluid Mechanics, 6th ed. (Academic Press, San Diego, 2012).

[11] F. Toschi and E. Bodenschatz, Lagrangian properties of particles in turbulence, Annu. Rev. Fluid Mech. 41, 375 (2009).

[12] Intergovernmental Panel on Climate Change and T. Stocker, Climate Change 2013: The Physical Science Basis: Working Group I Contribution to the Fifth Assessment Report of the Intergovernmental Panel on Climate Change (Cambridge University Press, Cambridge, 2014).

[13] G. Falkovich, A. Fouxon, and M. G. Stepanov, Acceleration of rain initiation by cloud turbulence, Nature (London) 419, 151 (2002).

[14] A. Celani, G. Falkovich, A. Mazzino, and A. Seminara, Droplet condensation in turbulent flows, Europhys. Lett. 70, 775 (2005).

[15] A. Celani, A. Mazzino, and M. Tizzi, The equivalent size of cloud condensation nuclei, New J. Phys. 10, 075021 (2008).

[16] I. Fouxon and A. Leshansky, Phytoplankton's motion in turbulent ocean, Phys. Rev. E 92, 013017 (2015).

[17] A. Bracco, P. H. Chavanis, A. Provenzale, and E. A. Spiegel, Particle aggregation in a turbulent Keplerian flow, Phys. Fluids 11, 2280 (1999).

[18] M. Wilkinson, B. Mehlig, and V. Uski, Stokes trapping and planet formation, Astrophys. J. Suppl. Ser. 176, 484 (2008).

[19] B. T. Chao, Turbulent transport behavior of small particles in dilute suspension, Österreichisches Ingenieur-Archiv 18, 7 (1964).

[20] C. M. Tchen, Mean value and correlation problems connected with the motion of small particles suspended in a turbulent fluid, Ph.D. thesis, University of Delft, 1947.

[21] J. O. Hinze, Turbulence (McGraw-Hill, New York, 1975).

[22] R. Mei, R. J. Adrian, and T. J. Hanratty, Particle dispersion in isotropic turbulence under Stokes drag and Basset force with gravitational settling, J. Fluid Mech. 225, 481 (1991).

[23] L. M. Pismen and A. Nir, On the motion of suspended particles in stationary homogeneous turbulence, J. Fluid Mech. 84, 193 (1978).

[24] M. W. Reeks, On the dispersion of small particles suspended in an isotropic turbulent fluid, J. Fluid Mech. 83, 529 (1977).

[25] M. W. Reeks, The relationship between Brownian motion and the random motion of small particles in a turbulent flow, Phys. Fluids 31, 1314 (1988).

[26] L.-P. Wang and D. E. Stock, Dispersion of heavy particles by turbulent motion, J. Atmos. Sci. 50, 1897 (1993).

[27] L. P. Corgnati, C. Mantovani, A. Griffa, M. Berta, P. Penna, P. Celentano, L. Bellomo, D. F. Carlson, and R. D'Adamo, Implementation and validation of the ISMAR high-frequency coastal radar network in the Gulf of Manfredonia (Mediterranean Sea), IEEE J. Oceanic Eng. (2018), doi: 10.1109/JOE.2018.2822518.

[28] A. Mazzino and M. Vergassola, Interference between turbulent and molecular diffusion, Europhys. Lett. 37, 535 (1997). 
[29] M. R. Maxey and J. J. Riley, Equation of motion for a small rigid sphere in a nonuniform flow, Phys. Fluids 26, 883 (1983).

[30] T. R. Auton, Dynamics of bubbles, drops, and particles in motion in liquids, Ph.D. thesis, University of Cambridge, Cambridge, UK, 1984.

[31] T. R. Auton, J. C. R. Hunt, and M. Prud'Homme, The force exerted on a body in inviscid unsteady non-uniform rotational flow, J. Fluid Mech. 197, 241 (1988).

[32] E. E. Michaelides, Hydrodynamic force and heat/mass transfer from particles, bubbles, and drops-The Freeman Scholar Lecture, J. Fluids Eng. 125, 209 (2003).

[33] U. Marini Bettolo Marconi, A. Puglisi, L. Rondoni, and A. Vulpiani, Fluctuation-dissipation: Response theory in statistical physics, Phys. Rep. 461, 111 (2008).

[34] F. Candelier, J. Einarsson, and B. Mehlig, Angular Dynamics of A Small Particle in Turbulence, Phys. Rev. Lett. 117, 204501 (2016).

[35] P. Castiglione, A. Mazzino, P. Muratore-Ginanneschi, and A. Vulpiani, On strong anomalous diffusion, Phys. D (Amsterdam, Neth.) 134, 75 (1999).

[36] G. Biroli, A crash course on ageing, J. Stat. Mech. (2005) P05014.

[37] G. Falkovich, K. Gawędzki and M. Vergassola, Particles and fields in fluid turbulence, Rev. Mod. Phys. 73, 913 (2001).

[38] R. Kubo, M. Toda, and N. Hashitsume, Statistical Physics II: Nonequilibrium Statistical Mechanics (Springer-Verlag, Berlin, 1991).

[39] M. R. Maxey, The motion of small spherical particles in a cellular flow field, Phys. Fluids 30, 1915 (1987).

[40] M. Farazmand and G. Haller, The Maxey-Riley equation: Existence, uniqueness, and regularity of solutions, Nonlinear Anal.: Real World Appl. 22, 98 (2015).

[41] M. Martins Afonso, A. Mazzino, and P. Muratore-Ginanneschi, Eddy diffusivities of inertial particles under gravity, J. Fluid Mech. 694, 426 (2012).

[42] L. Bergougnoux, G. Bouchet, D. Lopez, and E. Guazzelli, The motion of solid spherical particles falling in a cellular flow field at low Stokes number, Phys. Fluids 26, 093302 (2014).

[43] R. Czopnik and P. Garbaczewski, Brownian motion in a magnetic field, Phys. Rev. E 63, 021105 (2001).

[44] B. Kursunoglu, Brownian motion in a magnetic field, Ann. Phys. 17, 259 (1962).

[45] D. S. Lemons and D. L. Kaufman, Brownian motion of a charged particle in a magnetic field, IEEE Trans. Plasma Sci. 27, 1288 (1999).

[46] P. S. Alekseev, Negative Magnetoresistance in Viscous Flow of Two-Dimensional Electrons, Phys. Rev. Lett. 117, 166601 (2016).

[47] W. B. Russel, Brownian motion of small particles suspended in liquids, Annu. Rev. Fluid Mech. 13, 425 (1981).

[48] L. E. Reichl, A Modern Course in Statistical Physics, 4th ed. (Wiley, New York, 2016).

[49] O. A. Druzhinin and L. A. Ostrovsky, The influence of Basset force on particle dynamics in twodimensional flows, Phys. D (Amsterdam, Neth.) 76, 34 (1994).

[50] S. Boi, M. Martins Afonso, and A. Mazzino, Anomalous diffusion of inertial particles in random parallel flows: Theory and numerics face to face, J. Stat. Mech.: Theor. Exp. (2015) P10023.

[51] P. Monroy, E. Hernandez-Garcia, V. Rossi, and C. Lopez, Modeling the dynamical sinking of biogenic particles in oceanic flow, Nonlin. Processes Geophys. 24, 293 (2017).

[52] R. Gatignol, The Faxén formulas for a rigid particle in an unsteady non-uniform Stokes-flow, J. Mec. Theor. Appl. 2, 143 (1983).

[53] S. Boi, A. Mazzino, and P. Muratore-Ginanneschi, Eddy diffusivities of inertial particles in random Gaussian flows, Phys. Rev. Fluids 2, 014602 (2017).

[54] P. G. Saffman, The lift on a small sphere in a slow shear flow, J. Fluid Mech. 22, 385 (1965).

[55] P. G. Saffman, Corrigendum to "The lift on a small sphere in a slow shear flow," J. Fluid Mech. 31, 624 (1968).

[56] K. L. Henderson, D. R. Gwynllyw, and C. F. Barenghi, Particle tracking in Taylor-Couette flow, Eur. J. Mech. B/Fluids 26, 738 (2007).

[57] D. Legendre and J. Magnaudet, The lift force on a spherical bubble in a viscous linear shear flow, J. Fluid Mech. 368, 81 (1998). 
[58] A. Tomiyama, Struggle with computational bubble dynamics, Multiphase Sci. Technol. 10, 369 (1998).

[59] D. Leighton and A. Acrivos, The lift on a small sphere touching a plane in the presence of a simple shear flow, J. App. Math. Phys. 36, 174 (1985).

[60] R. Mei, An approximate expression for the shear lift force on a spherical particle at finite Reynolds number, Int. J. Multiphase Flow 18, 145 (1992).

[61] E. S. Asmolov and J. B. McLaughlin, The inertial lift on an oscillating sphere in a linear shear flow, Int. J. Multiphase Flow 25, 739 (1999).

[62] N. Lukerchenko, Y. Kvurt, I. Keita, Z. Chara, and P. Vlasak, Drag force, drag torque, and Magnus force coefficients of rotating spherical particle moving in fluid, Part. Sci. Technol. 30, 55 (2012). 